\title{
A Method Of Automatic Trend Identification On Frequency
}

\author{
Fan Yang \\ School of Electrical and Electronic Engineering,North China Electric Power University (Baoding), \\ Baoding 071000, China \\ 2632460413@qq.com
}

Keywords: frequency trend, automatic identification, data smoothing, trend analysis, power system, WAMS

\begin{abstract}
Based on the measured data of wide area measurement system(WAMS),a method of automatic trend identification for frequency in power system is proposed.Using data-smoothing technology to eliminate the influence of the signal noise such as burr to extract the overall trend of the signal and combining with the technology of trend analysis,the automatic identification of the frequency curve is realized by the adaptive algorithm. The method overcomes the shortcomings of the traditional method of hard threshold, which is easy to be disturbed by the noise and has difficulty in setting threshold.At the same time, the calculating speed of the method is high enough to be used for on-line analysis and helping plan dispatching the electric power system. The experimental results show the effectiveness of the proposed method.
\end{abstract}

\section{Introduction}

Due to the influence of load fluctuation, system failure, and many other factors, the power system during operation will inevitably be disturbed.After the disturbance to power system occurs, We should get access to the scene of system disturbance timely and accurately and identify the type and location of the disturbance,in order to implement reasonable and effective control measures to safeguard the security and stable operation of the power grid.WAMS is a kind of new generation of network-wide monitoring system with synchronized phasor measurement unit PMU as the basic element[1-3],which has the ability to achieve the real-time network-wide synchronization of data acquisition,real-time as well as long-distance transmission in order to make the whole network dynamic monitoring possible.PMU throughout the whole network will put the collected information into the dispatching center in real time when the disturbance occurs. And then the operators can process and filter the real-time data to obtain valid information characterizing the features of the disturbance,as well as categorize the disturbance to determine the next step to cope.However, the automatic recognition is still very difficult when the disturbance occurs.In existing recognition programs of disturbance occurring, the method of threshold determination is mainly used.

The conventional method of frequency fluctuation is calculated as follows:

$$
\Delta f=f(t)-f(t-5)
$$

Due to the non-mutations of frequency,and based on experience, the trend of the frequency change caused by the disturbance can be reflected by the deviation between the value of the frequency in the 5 th cycle after the occurrence of the disturbance and the normal operational frequency which is $50 \mathrm{~Hz}$. In the equation (1),it represents a increase in the frequency when the deviation is positive,and shows a frequency drop when negative.

Through setting different threshold to different kinds of measured value,it is regarded that the disturbance occurs in the running system when measured value exceeds the threshold value we have set. Although the aforementioned technical proposal has high computing speed, its disadvantages can not be ignored as described below:

(1) Because of the disturbance of noise to the information in PMU measurement,sometimes the measured value exceeds the threshold simply caused by the noise.So the misjudgment of hard threshold method occurs timely. 
(2) It is very difficult to set a reasonable threshold due to the adjustment of grid operation mode, so the threshold should be adjusted accordingly.

(3)All the information of PMU measurement can reflect the disturbing feature.However the influence to different PMU sub-stations caused by the disturbance ought to be different due to their different positions. It is evident that existing method of threshold determination is unreasonable.

Based on the measured data of WAMS, in combination with artificial recognition of tendency with historical experience,as well as using the technology of trend analysis to design the algorithm of frequency identification[4], the trend of frequency can be automatically identified.

\section{Data Preprocessing}

Because the original data has a high frequency to produce and meantime there exists the noise interference,we need to preprocess the original data firstly in order to reduce the complexity of automatic identification. The basic idea is that an average value is extracted as an equivalent data in the time interval to simplify the data at each time interval.Given that the relative variation range of the extracted data is still large,the moving average processing rule is adopted,so that the trend of change can be obtained.Specific steps of the algorithm are as follows:

(1)Receive the original data $D t$ in real time and fix the time interval $T$, extract each time an average value as the time period of an equivalent data and save it in the variable $A$;

(2)Smoothing the equivalent data $A$ with the moving average method.Set the current data for $A(i)$ and forward shift amount for $D$,so the specific calculation formula is as follows,and the smoothed data is stored in the variable $B$;

$$
B(i)=\frac{\sum_{j=0}^{T-1} A(i-j)}{D}
$$

(3)Expand and fill with the data B,extend each data in B to a data segment of length T,then get the variable $\mathrm{C}$ and return.

\section{Frequency Trend Identification}

The method is to analyze and extract features from the frequency signal,and then identify the overall trend.According to the characteristic of the frequency signal,its fluctuation range is usually small,and generally a large oscillation does not occur, however,which is common in voltage signal.By judging the relationship between the change of the average value of the frequency in the interval and the threshold value, the result of trend recognition is given.The results of identification on the trend of frequency change are divided into three categories:steady, rise and fall.

The trend is identified by using the frequency information after pretreatment.The specific algorithm is as follows:

(1)Parameter initialization:the amount of historical data $k$,sampling interval $D$,threshold thre;

(2)Enter the original data and set the smoothed data for $B$,the Smoothing method refer to data preprocessing steps 1 ) to 3 );

(3)Data $B(j)$ and the front $\mathrm{k}-1$ data is processed to obtain the equation:

$$
\mathrm{D}(j)=\frac{1}{2}(\max (B(j: j-k+1))+\min (B(j: j-k+1)))
$$

(4) (4)Get the weighted average of the data $D(j)(j=1,2 \ldots k)$ to obtain the index C:

$$
C=\sum_{k=1}^{A} w(i) D(i-k)
$$

Where the weight meet $w(j-k) \leq w(j-(k-1)) \leq \mathrm{K} \leq w(j-1)$.

(5)Automatic trend recognition: 


$$
\begin{aligned}
& \text { if abs }(D(j)-C)>\text { thre } \\
& \text { if } D(j)<C \text { Down } \\
& \text { else UP } \\
& \text { end } \\
& \text { else }
\end{aligned}
$$

Normal

end

It should be noted that the value of the relevant parameters will also affect the recognition results in the specific implementation process:

(1)Value $T$ is the threshold value, the smaller the value is,the more part of the rise and fall will be found. The general recommendations are between $6 \sim 10$.

(2)Value $k$,length of historical data,is also important.The greater the value of $k$ is, the more close the link with the historical data will be.Taking account that the trend could be reasonably determined,the value of $k$ ought to be about 160 generally.

(3)In a time interval the extracts coming from a continuous $D$ raw data are processed into a data each time to smooth data and eliminate the burr. The volume $D$ will affect the accuracy of the judging result and the time delay. Because the change of the frequency data is not tremendous, the value $D$ is suggest to be about 5 .

\section{Case Analysis}

In this paper, according to the measured data of Shaanxi provincial power grid as an example, the proposed method is tested. The frequency signal measured by WAMS is showed in figure 1 .

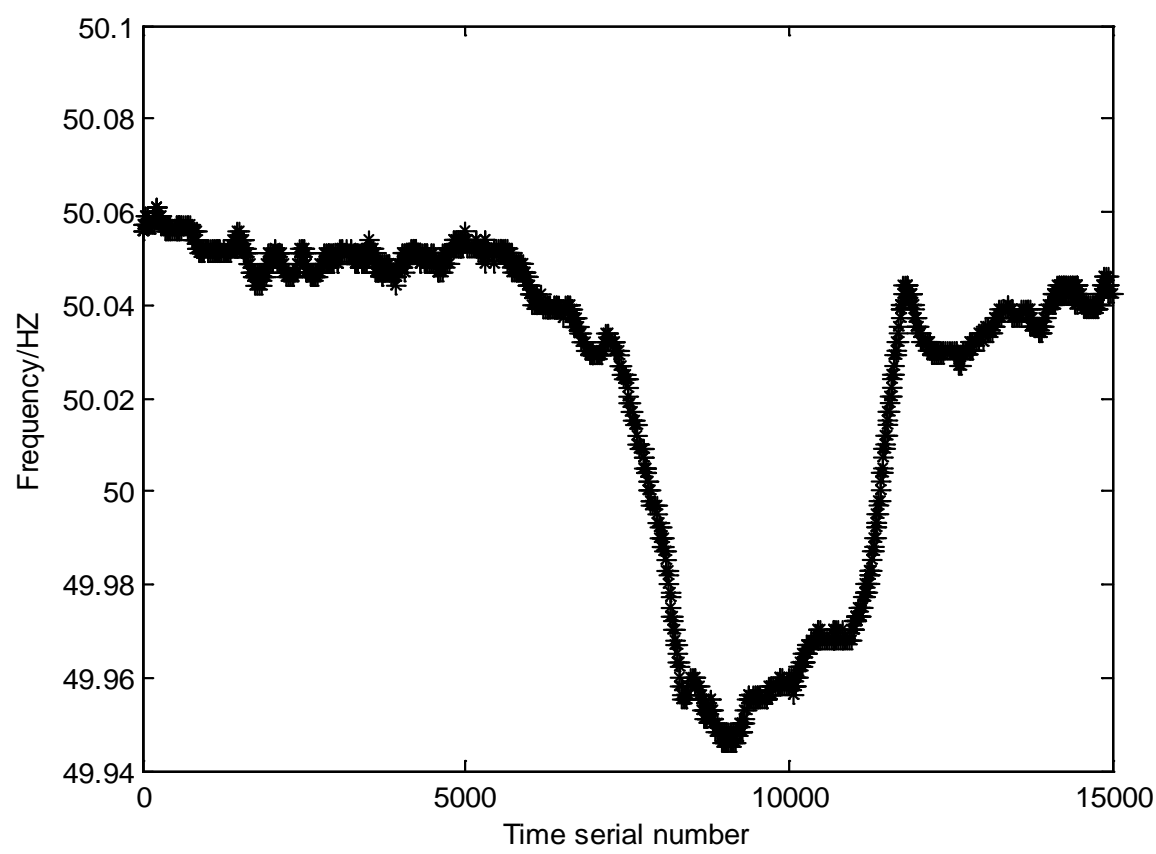

Fig 1 the measured data

And then Matlab programming is used to achieve the method to judge the trend of frequency signal in figure 1 . We set that $T=8.5, k=150, D=5$. And the effect of judgment is shown clearly in figure 2,in which the green line indicates a declining trend, and the red line indicates the rising trend.It is evident that the method proposed in this paper can effectively judge the change of the frequency trend. 


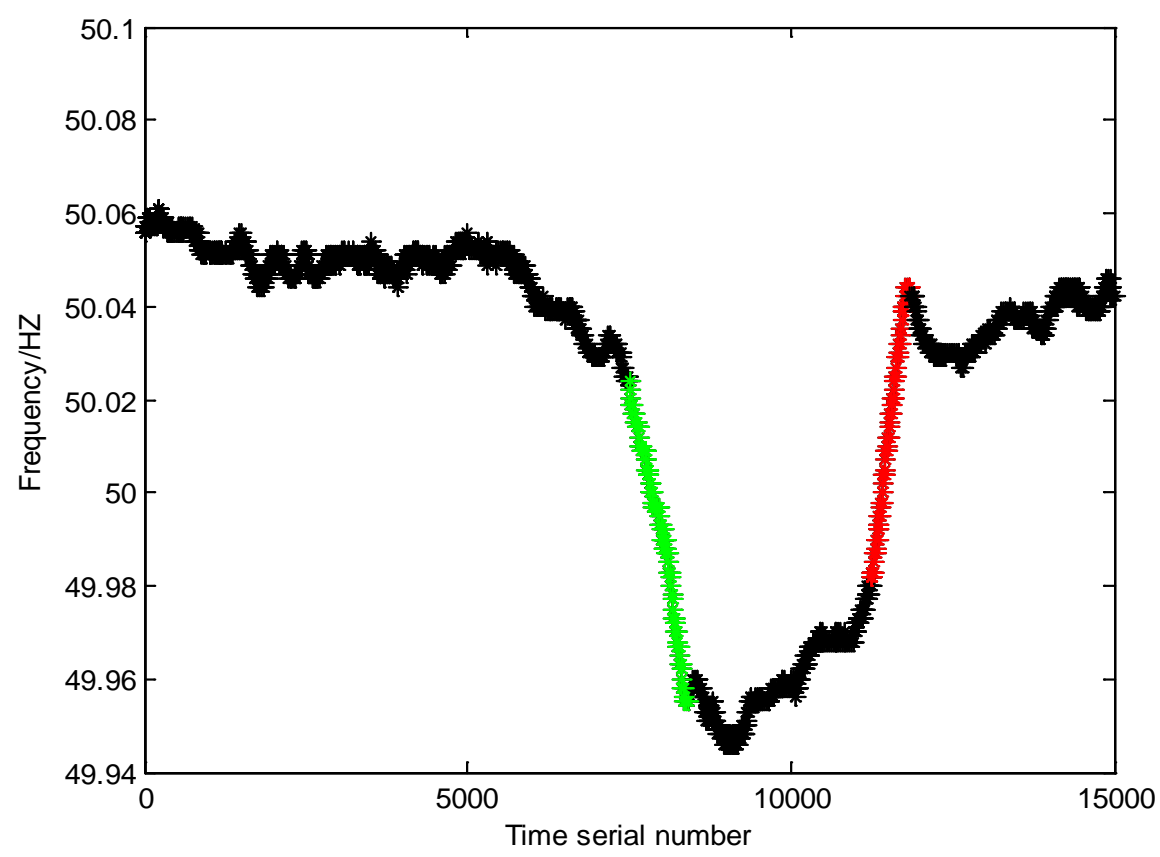

Fig 2 the effect of judgment

\section{Summary}

Based on the measured data of wide area measurement system,a method of automatic identification for frequency trend of power system is proposed. Using the processing technology of data smoothing to eliminate the influence of the signal noise to extract the overall trend of the signal and combining with the technology of trend analysis, the automatic identification of the frequency trend is realized by the adaptive algorithm. The method overcomes the shortcomings of the traditional means to set hard threshold, which is easy to be disturbed by the noise and meanwhile has difficulty in setting threshold. What's more, the calculation speed is high enough to be used for on-line analysis to help improve the security of grid operation.

\section{Acknowledgments}

This paper is funded by the University Students' Innovative Training Project.Finally,I would like to express my heartfelt thanks to Wang Tao and other teachers coming from Institute of mathematics and science, North China Electric Power University (Baoding)!

\section{References}

[1] Gang Duan,Yaqin Yan,Xiaodong Xie, Development Status Quo and Tendency of Wide Area Phasor Measuring Technology, J. Automation of Electric Power Systems. 2015, (1):73-80.

[2] Yusheng Xue, Haifeng Li,A Review of CIGRE 2008 on Power System Operation and Control, J. HIGH VOLTAGE ENGINEERING. 2008, 34(11):2253-2258.

[3] Yunfeng Cheng,Xinran Zhang,Chao Lu,Research progress of the application of wide area measurement technology in power system, J. Power System Protection and Control.2014,(4): 145-153.

[4] Qi Lei,Min Wu,Jinhua She,Trend analysis based fuzzy operating-state identification for combustion process of coke ovens,J. Chinese High Technology Letters.2011, 21(8): 879-885. 
